\title{
Diversidade e uso de hábitat por anfíbios anuros em duas localidades de Mata Atlântica, no norte do estado da Bahia
}

\author{
Flora Acuña Juncá \\ Biota Neotropica v6 (n2)-http://www.biotaneotropica.org.br/v6n2/pt/abstract?inventory+bn03006022006 \\ Recebido em 17/08/05. \\ Versão Reformulada recebida em 02/05/2006 \\ Publicado: em 20/06/2006
}

Depto. Ciências Biológicas, Universidade Estadual de Feira de Santana, Campus Universitário, BR 116, km 3, Feira de Santana, Bahia, 44.031-460, Brasil (fjunca@uefs.br)

\begin{abstract}
Juncá, F.A. Anuran diversity and habitat use in two localities of Atlantic forest in north of Bahia state. Biota Neotrop. May/

Aug 2006 vol. 6 no. 2, http://www.biotaneotropica.org.br/v6n2/pt/abstract?inventory+bn03006022006. ISSN 1676-0603

The composition and abundance of anurofauna, as well as its distribution among reproductive sites in two localities of Atlantic forest in northern Bahia state were studied. Serra da Jibóia (SJ) is a mountain range with maximum elevation of $800 \mathrm{~m}$, located in a transitional area between Atlantic forest and Caatinga. Reserva Sapiranga (RS) is a fragment of forest with $200 \mathrm{~m}$ of elevation and located close to the coast. In both localities, we searched for frogs in the following habitats: leaf litter/ understory, bromeliads, temporary ponds, permanent ponds and streams. Nocturnal collections were carried out through 10 months of 2001, totalizing 14 nights for RS and 12 nights for SJ. A total of 45 species were found, from which only seven were common to both localities. In SJ, more species were associated with the leaf litter/understory $(n=14)$ than the other reproductive sites, including two of the three more abundant species (Frostius penambucensis and Eleutherodactylus ramagii). In RS, temporary ponds were the reproductive sites that had greater species number $(\mathrm{n}=18)$, also including the two of the most abundant species (Leptodactylus natalensis and Scinax sp. ruber group). These differences could be related to differences in elevation, vegetation and leaf litter conditions of Serra da Jibóia and Reserva Sapiranga. In this study, we extend the geographic distribution of Leptodactylus mystaceus, Eleutherodactylus bilineatus, E. vinhai, Sphaenorhynchus prasinus, Phyllodytes melanomystax.
\end{abstract}

Key words: Anura, communities, spatial distribution, reproductive sites, conservation

\section{Resumo}

Juncá, F.A. Diversidade e uso de habitat por anfíbios anuros em duas localidades de Mata Atlântica, no norte do estado da

Bahia. Biota Neotrop. May/Aug 2006 vol. 6 no. 2, http://www.biotaneotropica.org.br/v6n2/pt/ abstract?inventory+bn03006022006. ISSN 1676-0603

A composição e abundância da anurofauna, bem como a distribuição das espécies em locais de reprodução, foram determinados em duas localidades na Mata Atlântica, norte do estado da Bahia. A Serra da Jibóia (SJ) é um maciço montanhoso com altitude máxima de 800 m, localizada em uma área de transição entre Caatinga e Mata Atlântica. A Reserva Sapiranga (RS) é um fragmento de mata a $200 \mathrm{~m}$ de altitude e localizado próximo ao litoral. Nas duas localidades, os seguintes hábitats foram amostrados: folhedo/sub-bosque, bromélias terrícolas, poças temporárias, poças permanentes e riachos. Ao longo de 10 meses de 2001, foram realizadas coletas noturnas, totalizando 14 noites de amostragem na Reserva Sapiranga (RS) e 12 na Serra da Jibóia (SJ). Um total 45 espécies foram registradas, sendo que apenas sete foram comuns às duas localidades. Na SJ, mais espécies $(\mathrm{n}=14)$ estiveram associadas ao folhedo/sub-bosque do que aos outros sítios reprodutivos, incluindo Frostius pernambusencis e Eleutherodactylus ramagii, duas das três espécies mais abundantes nessa localidade. Na RS, as poças temporárias foram os hábitats que apresentaram maior número de espécies $(\mathrm{n}=18)$, incluindo também as duas mais abundantes (Leptodactylus natalensis e Scinax sp. grupo ruber) nessa localidade. Estas diferenças podem ser atribuídas as diferenças de altitude, vegetação e condições do folhedo nas duas localidades. Neste estudo foi registrado o aumento na área de distribuição de Leptodactylus mystaceus, Eleutherodactylus bilinetus, E. vinhai, Sphaenorhynchus prasinus e Phyllodytes melanomystax.

Palavras-chave: Anura, comunidades, distribuição espacial, locais de reprodução, conservação 


\section{Introdução}

A importância da Mata Atlântica como uma das florestas mais diversificadas em organismos e, ao mesmo tempo, ameaçada por extensos desmatamentos que remontam desde o século XVI, tem sido amplamente noticiada (para revisão ver Galindo-Leal \& Câmara 2005). Neste bioma altamente fragmentado, ocorrem cerca de 340 espécies de anuros, que correspondem à cerca de 20\% das espécies da América do Sul (Haddad \& Abe 1999). Esta riqueza é acompanhada por uma elevada porcentagem de espécies endêmicas, muitas delas com distribuição restrita a uma determinada região ou microrregião da Mata Atlântica (Haddad \& Abe 1999, Duellman 1999). De fato, uma parcela significativa das espécies endêmicas da Mata Atlântica é conhecida apenas de sua localidade tipo (Haddad \& Abe 1999), e a carência de informações sobre estas espécies pode estar induzindo uma avaliação incorreta de sua real distribuição e, por conseguinte, de seu endemismo.

Por outro lado, casos de extinção e declínio populacional de diferentes espécies de anuros foram notados em diferentes continentes (Pechmann \& Wilbur 1994, Stebbins \& Cohen 1995, Halliday 1998; Pounds et al. 1999). Na Mata Atlântica, embora tenham sido registrados declínio e desaparecimento de espécies antes abundantes (Heyer et al. 1988, Bertoluci \& Heyer 1995, Eterovick et al. 2005), é extremamente difícil julgar o grau de ameaça às espécies, principalmente frente ao desconhecimento sobre a distribuição, uso de hábitat e biologia das fases larval e adulta das espécies (Young et al. 2001).

Trabalhos mais intensivos, com amostragens freqüentes e realizadas ao menos ao longo de um ano, enfocando a estrutura e padrões de abundância da comunidade, ocorrência sazonal e uso do hábitat pelas espécies de anuros em áreas de Mata Atlântica são raros, pontuais e realizados principalmente na região sudeste (Cardoso et al. 1989, Heyer et al. 1990, Haddad \& Sazima 1992, Arzabe et al. 1998, Bertoluci 1998, Bertoluci 2001, Bertoluci \& Rodrigues, 2002a, b, Silvano \& Pimenta 2003). Assim, é urgente a realização de mais estudos que permitam reconhecer o real status de conservação das diferentes espécies que ocorrem neste bioma.

A maioria dos estudos sobre a anurofauna da Mata Atlântica no estado da Bahia tem se restringido a descrições de espécies, principalmente no sul do estado (para revisão, ver Silvano \& Pimenta 2003). Entretanto, pouco se conhece sobre a composição de espécies das comunidades de anuros da mata atlântica do Recôncavo Baiano e do Litoral Norte do estado da Bahia. Este trabalho teve como objetivo inventariar as espécies de anuros em duas localidades distintas de Mata Atlântica, situadas respectivamente ao norte e oeste de Salvador, bem como estudar a riqueza, a abundância das espécies e a sua distribuição nos hábitats disponíveis para reprodução.

\section{Material e Métodos}

Durante o ano de 2001, na Serra da Jibóia e na Reserva Sapiranga foram realizadas quatro (abril, julho, setembro, e dezembro) e cinco excursões (março, maio, julho, setembro e novembro), respectivamente, cada uma com duração de duas a quatro noites. Na Reserva Sapiranga, foi realizado um total de 14 noites de observação e mais uma noite na Fazenda Camurugipe (área vizinha à Reserva Sapiranga), enquanto que na Serra da Jibóia foram realizadas 12 noites de observação. O número de observadores variou de dois a quatro. As atividades de campo iniciavam antes do anoitecer (17:30 h) e finalizavam quando todos os hábitats tivessem sido amostrados.

\section{1. Áreas de estudo e hábitats amostrados 1.1 Serra da Jibóia (SJ)}

A Serra da Jibóia está localizada entre os municípios de Santa Terezinha e Elísio Medrado e pertence a um conjunto de serras disjuntas que se estendem desde o litoral sul do estado da Bahia em direção noroeste e norte até a região da Baia de Todos os Santos (Figura 1). A altitude máxima alcança aproximadamente $800 \mathrm{~m}$. Situada entre os domínios de Mata Atlântica e Caatinga sensu Ab’Saber (1977), é um dos pontos mais a oeste da mata atlântica baiana e uma das matas úmidas de encosta situada mais ao norte do estado. Essa localização e altitude proporcionam extensas áreas de caatinga no lado oeste da serra, mata ombrófila úmida densa ao leste e, nos cumes, uma vegetação de campo rupestre. Por toda a Serra, encontram-se pequenas e médias propriedades rurais com atividades agropecuárias. Alguns fragmentos de mata secundária ainda são encontrados.

Um total de cinco tipos de hábitats potenciais para reprodução de anuros (Tabela 1) foram amostrados na mata e no campo rupestre na Pioneira (denominação de uma das montanhas da Serra da Jibóia, com altitude de aproximadamente 800 m), distrito de Pedra Branca, município de Santa Terezinha, e na Reserva Jequitibá, uma RPPN localizada também na Serra da Jibóia, no município de Elísio Medrado:

Folhedo. O folhedo de mata é muito abundante e úmido, enquanto que o folhedo de campo rupestre está restrito às proximidades de alguns arbustos, permanecendo mais exposto ao sol e, portanto, menos úmido.

Bromélias terrícolas. Estão presentes no campo rupestre e na mata e são distintas nos dois ambientes. Aquelas do campo rupestre são bem maiores e ocorrem em maior densidade que as bromélias da mata (Juncá \& Borges 2002). Ao menos três espécies de bromélias foram identificadas: Alcantaraea extensa (campo rupestre), Vrisea noblickii e Nidularium sp. (ambas de mata).

Poças temporárias. As poças temporárias observadas na Pioneira e na Reserva Jequitibá são formadas nas margens de riachos, possuindo fundo lodoso e vegetação constituída 
por gramíneas, ciperáceas, arbustos e, em toda sua extensão, algumas árvores emergentes com mais de $30 \mathrm{~m}$ de altura. Foram observadas com água apenas durante o mês de junho.

Riachos. Dentro da mata, são encontrados riachos que variam entre 2 a $4 \mathrm{~m}$ de largura, levemente encachoeirados e com fundo que se alterna entre trechos pedregosos (com maior velocidade da água) e trechos arenosos (com menor velocidade da água). A vegetação é densa nas margens, como nas poças temporárias. Paredões rochosos também foram observados, mas não foram amostrados.

Poças permanentes. São alimentadas por córregos e apresentam fundo arenoso ou lodoso. A vegetação marginal é semelhante à dos ambientes aquáticos acima descritos. Também foi amostrado um tanque de alvenaria, que provavelmente acumula água da chuva, com $3 \mathrm{~m}^{2} \mathrm{de}$ área e $1 \mathrm{~m}$ de profundidade, distando aproximadamente $10 \mathrm{~m}$ da borda do campo rupestre da Pioneira, com grande quantidade de algas em suas paredes, onde foram observados girinos. Este tanque apresentou água durante todos os meses.

\subsection{Reserva Sapiranga (RS)}

A Reserva Sapiranga é uma RPPN com cerca de 600 hectares, localizada em frente à Praia do Forte (Município de Mata de São João), litoral norte da Bahia, a aproximadamente $50 \mathrm{~km}$ de Salvador, com altitude média de 200 m (Figura 1). A reserva está localizada em uma área de transição entre restinga e mata, com solo na maioria das vezes arenoso, apresentando trechos de mata secundária em estado de regeneração, com árvores emergentes que podem alcançar entre 20 a 30 m de altura, e áreas abertas, onde predomina vegetação arbustiva. Trilhas sinuosas permeiam estas diferentes formações vegetais e são utilizadas por turistas. A oeste, e contínua à área da reserva, há uma área de mata também secundária, localizada na fazenda Camarujipe que, como a Reserva Sapiranga (RPPN), pertencente à Fundação Garcia D’ Avila. Foram identificados ao longo destas trilhas cinco diferentes hábitats potencialmente adequados para reprodução de anuros, similar a Serra da Jibóia:

Folhedo de mata que, comparado ao folhedo de mata da Serra da Jibóia, tem menor profundidade sendo, portanto, menos abundante, na maioria das áreas observadas.

Bromélias terrícolas. Somente em uma área de aproximadamente $2000 \mathrm{~m}^{2}$ dentro da mata foram observadas bromélias terrícolas, com uma densidade de indivíduos similar a observada no campo rupestre na Serra da Jibóia. As bromélias da Reserva Sapiranga não foram identificadas, mas sua arquitetura e dimensões foram similares às bromélias amostradas no campo rupestre da Serra da Jibóia.

Poças temporárias. Uma depressão dentro da mata e, portanto, cercada de árvores e sub-bosque, de fundo arenoso, reteve água somente no mês de setembro de 2001.
Ao longo da margem direita do rio Pojuca, foram observadas três poças cheias nos meses de março a julho de 2001. A vegetação ao redor dessas poças era escassa e constituída por gramíneas e alguns arbustos, que alcançavam até $5 \mathrm{~m}$. No mês de setembro de 2001, estas poças foram inundadas, devido a um aumento da vazão do rio Pojuca. No mês de novembro, as poças estavam secas ou com pouca água, porém eutrofizadas.

Poça permanente. O solo do fundo variou de arenoso a lodoso, e vegetação marginal predominante era arbustiva, de até $3 \mathrm{~m}$ da atura, distando mais ou menos $10 \mathrm{~m}$ da área com vegetação mais alta.

Riachos. Dois riachos afluentes do rio Pojuca: riacho Terebus, com profundidade máxima de $1 \mathrm{~m}$ e largura de $2 \mathrm{~m}$, e fundo arenoso; e riacho Sapiranga, com trechos de fundo arenoso e profundidade máxima de $0,5 \mathrm{~m}$, e trechos de fundo lodoso, com acúmulo de matéria orgânica, principalmente nas margens alagadas, durante os meses de setembro e novembro de 2001, quando a largura alcançou aproximadamente $3 \mathrm{~m}$. Em ambos os riachos, a vegetação era abundante nas margens, com gramíneas, vegetação arbustiva e árvores alcançando $20 \mathrm{~m}$.

Além da Reserva Sapiranga, também foi realizada uma coleta noturna em uma outra área de mata, na fazenda Camurugipe. Nesta área, foram observados um riacho, o Córrego do Rego, e uma lagoa permanente com aproximadamente $200 \mathrm{~m}^{2}$ de área, ambos no interior da mata.

\section{Amostragem dos hábitats}

Em cada localidade foram amostrados cinco tipos de hábitats: folhedo, riachos, poças temporárias, poças permanentes e bromélias (Tabela 1). Foram feitas transeções de diferentes comprimentos em decorrência dos diferentes tamanhos das áreas, especialmente a de campo rupestre na Serra da Jibóia e a área com bromélias terrícolas na Reserva Sapiranga, e comprimento dos trechos amostráveis dos riachos.

Nas áreas de mata (RS e SJ) e de campo rupestre (SJ) a abundância das espécies foi determinada por registro visual e acústico das espécies, ao longo das transeções realizadas nas áreas de folhedo e sub-bosque. Nas transeções na mata e campo rupestre da Serra da Jibóia, as bromélias foram vistoriadas, e as espécies registradas com base na vocalização e no encontro de adultos ou girinos. Na Reserva Sapiranga, ao longo das transeções, as bromélias foram muito escassas. Assim, foi amostrado um outro trecho de mata que apresentou bromélias com arquitetura e densidade similares ao do campo rupestre da Serra da Jibóia (tabela 1), utilizando-se o mesmo procedimento para a amostragem já descrito. As poças permanentes e temporárias (tabela 1) foram percorridas ao longo de seu perímetro, quando também foi estimado o número de indivíduos que 
vocalizavam de cada espécie e registrados os encontros visuais durante a procura ativa. Os riachos tiveram trechos amostrados (Tabela 1), onde as espécies foram registradas e estimadas as respectivas abundâncias através de procura ativa e estimativa do número de indivíduos que vocalizavam. Em cada noite de observação, a averiguação do número de indivíduos em cada um dos pontos de amostragem (transeções, poças e riachos) foi realizada em de 20 minutos.

Além das observações no campo, para completar a lista de espécies de cada localidade, foram utilizados os registros da Coleção de Animais Peçonhentos e Herpetologia do Museu de Zoologia da UEFS (MZUEFS), para as localidades em questão.

\section{Análises estatísticas}

A cada noite de observação, foi calculada a abundância de cada espécie, através da soma do número de adultos visualizados e da estimativa do número de indivíduos vocalmente ativos. Como abundância total de cada espécie, foi considerada a maior abundância obtida ao longo de todo o período estudado (maior número de indivíduos/noite) (Bertoluci \& Rodrigues 2002b, Vasconcelos \& Rossa-Feres 2005). A estimativa do número de indivíduos em vocalização é amplamente utilizada para anuros e tem sido indicada (Heyer et al. 1994) e avaliada como eficiente, além da diferença entre observadores ser considerada não significativa (Shirose et al. 1997).

Para verificar a eficiência da metodologia de amostragem, foi construída uma curva de rarefação para cada localidade, com 500 aleatorizações, geradas a partir da matriz de dados de abundância de cada noite de observação (RS = 15 amostragens; SJ = 12 amostragens). A riqueza de espécies foi estimada pelas equações de Jackknife de primeira e segunda ordem e Bootstrap. A curva de rarefação e estimativas de riqueza de espécies foram calculadas no programa computacional EstimateS versão 7.00 (Colwell 2004). O índice Jackknife estima a riqueza absoluta somando a riqueza observada a um parâmetro calculado a partir do número de espécies raras e do número de amostras, enquanto o método Bootstrap utiliza dados de todas as espécies amostradas, não se restringindo às raras (Santos 2003).

A identificação das espécies foi baseada nas descrições disponíveis na literatura, incluindo informações sobre canto de anúncio, e comparações com exemplares depositados no Museu Nacional da Universidade Federal do Rio de Janeiro e no Museu de Zoologia da Universidade de São Paulo. O material testemunho, incluindo as gravações de canto de anúncio, foi depositado na Coleção de Animais Peçonhentos e Herpetologia do Museu da Universidade Estadual de Feira de Santana (MZUEFS).

\section{Resultados}

Foram registradas 46 espécies de anuros, distribuídas em quatro famílias (Bufonidae, Hylidae, Leptodactylidae e Dendrobatidae), sendo que apenas sete (Bufo jimi, Leptodactylus ocellatus, Eleutherodactylus ramagii, Eleutherodactylus sp. grupo binotatus, Hypsiboas albomarginatus, Phyllodytes melanomystax e Scinax $x$ signatus) foram comuns às duas localidades amostradas: Reserva Sapiranga e Serra da Jibóia.

Na Reserva Sapiranga e fazenda Camurugipe, foram registradas 25 espécies (Tabela 1, Figura 2). Somente uma espécie, Bufo sp. grupo margaritifer, coletada na Reserva Sapiranga (MZUEFS 57), não foi encontrada durante o período de estudo. A maioria destas espécies foi registrada nas poças temporárias e permanentes. Dois hilídeos, Phyllodytes melanomystax (Figura 2d) e Scinax agilis foram observados nas bromélias e as duas espécies de Eleutherodactylus, no folhedo. Com exceção de Sphaenorhynchus prasinus, cuja observação foi pontual devido a uma única visita a fazenda Camarujipe, as demais espécies foram observadas nos locais de reprodução da Reserva Sapiranga. Seis destes locais foram monitorados em todas as excursões e um, poça permanente, foi amostrado apenas nos dois últimos meses, o que possibilitou o registro de Hypsiboas faber e Dendropsophus elegans. Apenas indivíduos recém metamorfoseados de Proceratophrys aff. boiei foram observados, indivíduos adultos não foram encontrados.

Na Serra da Jibóia, foram registradas 22 espécies (Tabela 2, Figura 3). Outras oito espécies não registradas no presente estudo são consideradas na lista dessa localidade, com base nos registros de exemplares depositados no MZUEFS (A. pachydactyla MZUEFS 45, 47, 48, 78; Crossodactylus sp. MZUEFS 1375, Odontophrynus carvalhoi MZUEFS 80; Gastrotheca fissipes MZUEFS 5, 296; Gastrotheca sp. MZUEFS 657; H. crepitans MZUEFS 52; Colostethus alagoanus MZUEFS 81). Diferente da Reserva Sapiranga, o folhedo da mata foi o ambiente que apresentou maior número de espécies, seguido pelas poças permanentes. Apenas uma espécie foi observada nas poças temporárias (Bokermannohyla hylax, Figura 3d) e uma no folhedo do campo rupestre (Eleutherodactylus ramagii).

A curva de rarefação obtida para a Reserva Sapiranga (Figura 4a) mostrou uma tendência a estabilização, culminando em um desvio padrão próximo a zero. Por outro lado, a curva obtida para a Serra da Jibóia (Figura 4b) indica a necessidade de um esforço amostral maior para registrar o número total de espécies desta localidade. As estimativas de riqueza para a Reserva Sapiranga ( $\mathrm{n}=15$ amostras) foram de 27,73 $\pm 1,65$ e 27,18 $\pm 0,00$ espécies, quando utilizado o estimador Jackknife (primeira e segunda ordem, respectivamente) e de 26,14 $\pm 0,56$ espécies quando utilizado Bootstrap. Na Serra da Jibóia ( $\mathrm{n}=12$ amostras) a riqueza estimada foi de 25,66 $\pm 1,56$ e 25,21 $\pm 2,98$ espécies, quando 
utilizado Jackknife (primeira e segunda ordem, respectivamente) e de 24,04 $\pm 0,66$ espécies, quando utilizado o estimador Bootstrap. Os valores de riqueza estimados pelos diferentes índices são próximos aos obtidos para as duas localidades quando se considera as espécies registradas nesse estudo e aquelas depositadas no MZUEFS ( $\mathrm{RS}=24$ espécies e $\mathrm{SJ}=30$ ).

As curvas do componente dominância (Figura 5) revelam que uma pequena proporção das espécies foi muito abundante (19\% na RS e 14\% na SJ), enquanto uma grande proporção apresentou abundância intermediária (33,3\% RS e 23\% SJ) ou baixa (33\% RS e $36 \%$ SJ) e poucas espécies foram raras (14,3\% RS e 27\% SJ). Na Serra da Jibóia (Figura 6a) as espécies mais abundantes foram Frostius pernambucensis, cujos machos foram ouvidos por toda a mata da serra, Bokermannohyla hylax e Eleutherodactylus ramagii. Na Reserva Sapiranga, as espécies mais abundantes foram L. natalensis (Figura 2a) e Scinax sp. (Figura 6b).

\section{Discussão}

\section{Composição e diversidade da anurofauna}

A riqueza de espécies da Reserva Sapiranga e da Serra da Jibóia são intermediárias quando comparadas a outras localidades de Mata Atlântica, cuja riqueza tem variado entre 17 e 65 espécies (Heyer et al. 1990, Arzabe et al. 1998, Bertoluci \& Rodrigues 2002a, Silvano \& Pimenta 2003). Entretanto, a avaliação da metodologia de amostragem e o pequeno número de espécies comuns às duas localidades amostradas, indicam que o número de espécies registrado pode estar subestimado. Por outro lado, apenas 17 espécies foram registradas na Floresta do Castro, Sergipe, uma localidade de Mata Atlântica ao norte da Reserva Sapiranga, com um esforço amostral de 24 noites ao longo de um ano (Arzabe et al. 1998). Entretanto, aquele estudo restringiu-se a ambientes aquáticos e, portanto, espécies ocorrentes no folhedo ou bromélias daquela área não foram amostradas.

A composição e distribuição espacial das espécies da Serra da Jibóia e da Reserva Sapiranga apresentaram diferenças notáveis. A diversidade de gêneros e famílias foi maior na Serra da Jibóia. Ainda nesta localidade, também foi encontrada uma maior riqueza de espécies da família Leptodactylidade. Além do pequeno número de espécies em comum, os ambientes com maior riqueza de espécies foram distintos nas duas localidades: folhedo e sub-bosque na Serra da Jibóia e poças temporárias na Reserva Sapiranga. Provavelmente, este resultado deve-se a fatores históricos durante a colonização da região pelas diferentes linhagens de anuros, e às particularidades dos micro-hábitats, condicionados às diferenças na paisagem das duas localidades, que incluem aspectos da vegetação, altitude e clima. As áreas florestadas da Serra da Jibóia apresentaram um sub-bosque mais rico em bromélias terrícolas e também um folhedo mais abundante que na Reserva Sapiranga. Estas diferenças poderiam explicar, em parte, a presença de Frostius pernabucensis, Adelophryne pachydactyla, Colostethus alagoanus e também de uma maior quantidade de espécies de Eleutherodactylus na Serra da Jibóia, pois estas espécies utilizam as bromélias terrícolas ou o folhedo da mata como locais para reprodução. Além disso, a Reserva Sapiranga está muito próxima à restinga, que poderia determinar uma menor umidade dentro das manchas de mata.

As curvas do componente dominância das duas localidades foram similares, e as espécies mais abundantes estiveram associadas a mais de um tipo de hábitat ou aos ambientes onde foi registrada a maior riqueza de espécies. Na Reserva Sapiranga, Scinax sp. foi observada apenas nas poças temporárias às margens do rio Pojuca e Leptodactylus natalensis foi observada em todos os ambientes aquáticos, exceto nas poças onde vocalizava Scinax sp. Na Serra da Jibóia duas das espécies mais abundantes estavam dispersas pelo folhedo e sub-bosque (Eleutherodactylus ramagii e Frostius pernambucensis), e a terceira (Bokermannohyla hylax) esteve presente em todos os ambientes aquáticos amostrados. Eleutherodactylus ramagii provavelmente deposita os ovos no folhedo da mata ou na base das bromélias, modo reprodutivo encontrado em outras espécies do gênero (Duellman \& Trueb 1986, Haddad \& Prado 2005), enquanto Frostius pernambucensis utiliza bromélias terrícolas da mata como sítio de oviposição e desenvolvimento dos girinos (Cruz \& Peixoto 1982, Juncá \& Borges 2002). Já Bokermannohyla hylax foi a única espécie observada nos ambientes aquáticos temporários, mas ocorreu também nos permanentes. Deve-se considerar que as espécies que se reproduzem de forma explosiva, ou seja, que se reproduzem apenas durante alguns dias do período favorável para reprodução e depois não vocalizam mais, normalmente ocorrem em grande abundância quando estão em atividade reprodutiva (Duellman \& Trueb 1986). Nenhuma das espécies registradas na Serra da Jibóia apresentou esse padrão reprodutivo. Entretanto, na Reserva Sapiranga, Scinax sp. parece apresentar esta estratégia reprodutiva, pois machos desta espécie foram encontrados vocalizando apenas durante os quatro dias de observação no mês de maio de 2001.

Na Serra da Jibóia, alguns anuros foram ouvidos vocalizando no dossel, mas não foi possível amostrar esse hábitat. A exemplo de duas espécies registradas neste estudo que, muito provavelmente, são habitantes de bromélias arborícolas (Gastrotheca sp. e Eleutherodactylus sp.), uma amostragem mais intensiva desse microhabitat provavelmente revelaria mais espécies especializadas. 


\section{Implicações sobre o conhecimento da distribuição das espécies de anuros para sua conservação}

Com a amostragem na Serra da Jibóia a área de distribuição de Phyllodytes melanomystax, Eleutherodactylus vinhai e E. bilineatus, conhecidas apenas para o sudeste da Bahia (Silvano \& Pimenta 2003, Frost 2004), foi ampliada. Duas outras espécies, também encontradas nesta localidade, Frostius pernambucensis e Thoropa miliaris tiveram recentemente a ampliação de suas distribuições registradas (Feio et al. 2000, Juncá \& Freitas 2001). Frostius pernambucensis era previamente conhecida apenas de duas localidades: Reserva de Dois Irmãos, Recife, PE, e Murici, AL, enquanto que a distribuição geográfica de Thoropa miliaris estendia-se do estado de São Paulo até o Espírito Santo.

Phyllodytes wuchereri foi recentemente revalidada e redescrita (Caramaschi et al. 2004), porém, com a sua distribuição restrita ao sul da Bahia. A identificação desta espécie a partir dos exemplares coletados na Serra da Jibóia foi realizada principalmente pela presença de uma faixa branca lateral (Figura 3e). No entanto, na população da Serra da Jibóia, os espécimes não apresentaram o mosqueado marrom nas superfícies dorsais do tronco, da coxa e tíbia, presente nas populações estudas por Caramaschi et al. (2004), podendo tratar-se, portanto, de uma variação interpopulacional ou mesmo de uma outra espécie. Assim, serão necessários estudos comparativos das populações do sul da Bahia com a população da Serra da Jibóia, abrangendo morfologia dos adultos e girinos e vocalização, para definir se estas populações devem ser consideradas como táxons distintos ou mantidas em $P$. wuchereri.

Bokermannohyla hylax pertence ao grupo de $B$. circumdata, e embora novas espécies tenham sido encontradas no estado da Bahia (Napoli \& Pimenta 2003, M. Napoli \& F.J. Juncá, dados não publicados), seu registro na área estudada permite reconhecer um aumento na área de distribuição desta espécie, registrada até agora apenas para a Mata Atlântica do Sudeste do Brasil (São Paulo e Rio de Janeiro) (Frost 2004).

A amostragem na Reserva Sapiranga, permitiu reconhecer um aumento na área de distribuição de Leptodactylus mystaceus, Phyllodytes melanomystax (discutido acima) e Sphaenorhynchus prasinus. A primeira tem distribuição disjunta e pode representar uma das espécies que indicam uma ligação pretérita entre a Mata Atlântica do nordeste do país e a floresta Amazônica. Sphaenorhynchus prasinus era conhecida apenas de sua localidade tipo, Ilhéus e Teixeira de Freitas (Silvano \& Pimenta 2003), ambos municípios do sul da Bahia. Além dessas, foram registradas Hypsiboas pombali (Reserva Sapiranga) e Aplastodiscus sibilatus (Serra da Jibóia), espécies recentemente descritas, cuja distribuição abrange as matas do norte da Bahia (Cruz et al. 2003, Caramaschi et al. 2004).
A composição da anurofauna da Serra da Jibóia e da Reserva Sapiranga indica claramente a influência das florestas do sul da Bahia e sudeste do Brasil, e daquelas em outros estados ao norte da Bahia. Dentre as sugestões de áreas prioritárias para conservação de répteis e anfíbios, publicadas pelo Ministério do Meio Ambiente (2002), está uma área caracterizada como "insuficientemente conhecida mas de provável importância biológica”, que inicia-se no litoral da Baía de Todos os Santos e estende-se a oeste, onde localiza-se a Serra da Jibóia. Os resultados deste estudo corroboram esta avaliação, diante do aumento de distribuição de cinco espécies e de três espécies novas (uma delas já descrita - Aplastodiscus sibilatus). Por outro lado, naquele documento, foi ignorada uma extensão de mais de 230 quilômetros de Mata Atlântica, desde Salvador até Mangue Seco, ponto mais ao norte do litoral do estado da Bahia. Os resultados deste trabalho, em termos de espécies recentemente descritas, aumento da área de distribuição de três espécies e cinco táxons sem identificação ao nível específico mostra a necessidade da realização de mais estudos sobre a anurofauna nesta faixa litorânea, com vistas à conservação.

\section{Agradecimentos}

A José P. Pombal Jr., Ulisses Caramaschi, Carlos Alberto Gonçalves Cruz, Oswaldo Peixoto e Marcelo Napoli, pelo auxílio na identificação das espécies. A Elbano Paschoal e Adriano Paiva pela logística na RPPN Jequitibá e na Reserva Sapiranga, respectivamente. A Maria Conceição Lago Carneiro, Alessandra Silva Santana, Flávia Maria Pires Lima, Adriana Araújo da Silva, Pollyana da Silva Magalhães e Eric Souza de Jesus pelo auxílio em campo. A Gilson Evaristo Iack Ximenes pela confecção do mapa e sugestões. A FAPESB e UEFS pelo financiamento do trabalho.

\section{Referências}

AB'SABER, A..N. 1977. Os domínios morfoclimáticos da América do Sul. Primeira Aproximação. Geomorfologia 53:1-52

ARZABE, C., CARVALHO, C.X. \& COSTA, M.A.G. 1998. Anuran assembleges in Castro forest ponds (Sergipe State, Brazil): comparative struture and calling activity patterns. Hepetol. J. 8:111-113.

BERTOLUCI, J. 1998. Annual patterns of breeding activity in Atlantic Rainforest anurans. J. Herpetol. 32:607-611.

BERTOLUCI, J. 2001. Anfíbios anuros. In Intervales (C. Leonel, ed.). Fundação para a Conservação e a Produção Florestal do estado de São Paulo. Secretaria do Estado de São Paulo, Secretaria do Estado Meio Ambiente. Governo do estado de São Paulo, São Paulo, p.157-167. 
BERTOLUCI, J. \& W.R. HEYER 1995. Boraceia Update. Froglog 14.

BERTOLUCI, J. \& RODRIGUES, M.T. 2002a. Utilização de habitats reprodutivos e micro-habitats de vocalização em uma taxocenose de anuros (Amphibia) na Mata Atlântica no Sudeste do Brasil. Pap. Avulsos Zool. 42:287-297.

BERTOLUCI, J. \& RODRIGUES, M.T. 2002b. Seasonal patterns of breeding activity of Atlantic Rainforest anurans at Boracéia, Southeastern Brazil. Amphibia-Reptilia 23:161-167.

CARAMASCHI, U., PIMENTA, B.V.S. \& FEIO, R.N. 2004. Nova espécie do grupo Hyla geographica Spix, 1824 da Floresta Atlântica, Brasil (Amphibia, Anura, Hylidae). Bol. Mus. Nac., Zool. 518:1-14.

CARDOSO, A.J., ANDRADE, G.V. \& HADDAD, C.F.B. 1989. Distribuição espacial em comunidades de anfíbios (Anura) no sudeste do Brasil. Rev. Bras. Biol. 49(1):241-249.

COLWELL, R.K. 2004. EstimateS 7.00: statistical estimation of species richness and shared species from samples. Disponível em: http://viceroy.eeb.uconn.edu/estimates (último acesso 14/03/2005).

CRUZ, C.A. \& PEIXOTO, O.L. 1982. Sobre a biologia de Atelopus pernambucensis Bokermann, 1962 (Amphibia, Anura, Bufonidae). Rev. Bras. Biol. 42(3):627-629.

CRUZ, C.A., PIMENTA, B.V.S. \& SILVANO, D.L. 2003. Duas novas espécies pertencentes ao complexo de Hyla albosignata Lutz \& Lutz, 1983, do leste do Brasil (Amphibia, Anura, Hylidae). Bol. Mus. Nac., Zool. 503:1-13.

DUELLMAN, W.E. 1999. Patterns of distribution of amphibian. A global perspective. Johns Hopkins University Press, Baltimore and London.

DUELLMAN, W.E. \& TRUEB, L. 1986. Biology of Amphibian. Mc Graw-Hill, New York.

ETEROVICK, P.C., CARNAVAL, A.C.O.Q., BORGESNOJOSA, D.M., SILVANO, D.L., SEGALLA, M.V. \& SAZIMA, I. 2005. Amphibian declines in Brazil: an overview. Biotropica 37:166-179.

FEIO, R. N., JUNCÁ, F.A. \& CARAMASCHI, U. 2000. Geographic Distribution - Thoropa miliaris. Herpetol. Rev. 31:252.

FROST, D.R. 2004. Amphibian species of the world: an online reference. Version 3.0. Eletronic data-base accessible at: http://research.amnh.org/herpetology/amphibia/ index.html. (último acesso 01/05/2006).

GALINDO-LEAL, C.G. \& CÂMARA, I.D. 2005. Mata Atlântica Biodiversidade, Ameaças e Perspectivas. Conservation International, Belo Horizonte.
HADDAD, C.F.B. \& SAZIMA, I. 1992. Anfíbios anuros da Serra do Japi. In História natural da Serra do Japi: ecologia e preservação de uma área florestal no Sudeste do Brasil (L.P.C. Morellato, org.). Editora Unicamp/FAPESP, Campinas, p.188-211.

HADDAD, C.F.B. \& ABE, A. 1999. Anfíbios e Répteis. In: Workshop Floresta Atlântica e Campos Sulinos. http:// www.bdt.org.br/workshop/mata.atlantica/BR/rp anfib. (último acesso 01/05/2006)

HADDAD, C.F.B. \& PRADO, C.P.A. 2005. Reproductive modes in frogs and their unexpected diversity in the Atlantic Forest of Brazil. Bioscience 55:207-217.

HALLIDAY, T. 1998. A declining amphibian conundrum. Nature 394:418-419.

HEYER, W.R., RAND, A.S., DA CRUZ, C.A.G \& PEIXOTO, O.L. 1988. Decimation, extinctions and colonization of frog populations in Southeast Brazil and their evolutionary implications. Biotropica 20:230-235.

HEYER, W.R., RAND, A.S., DA CRUZ, C.A.G., PEIXOTO, O.L. \& NELSON, C.E. 1990. Frogs of Boracéia. Arq. Zool. 34:231-410.

HEYER, W.R., DONNELY, M.D., MACDIARMID, R.W., HAYEK, L.C. \& FOSTER, M.S. 1994. Measuring and Monitoring Biological Diversity. Standard Methods for Amphibians. Smithsonian Institution Press, Washington and London.

JUNCÁ, F.A. \& FREITAS, M. 2001. Geographic distribution. Frostius pernambucensis. Herpetol. Rev. 32:270-271.

JUNCÁ, F.A. \& BORGES, C.L.S. 2002. Fauna associada a bromélias terrícolas da Serra da Jibóia. Sitientibus, sér. Ciências Biológicas, 2:73-81.

MINISTERIO DO MEIO AMBIENTE 2002. Biodiversidade Brasileira. Avaliação e identificação de áreas e ações prioritárias para conservação, utilização sustentável e repartição de benefícios da biodiversidade nos biomas brasileiros. MMA, Secretaria de Biodiversidade e Florestas, Brasília.

NAPOLI, M.F. \& PIMENTA, B.V.S. 2003. Nova espécie do grupo de Hyla circumdata (Cope, 1870) do sul da Bahia, Brasil (Amphibia, Anura, Hylidae). Arq. Mus. Nac. 61:189194.

PECHMANN, J.H.K. \& WILBUR, H.M. 1994. Putting declining amphibian population in perspective: natural fluctuations and human impacts. Herpetologica 50:65-84.

POUNDS, J.A., FOGDEN, M.P.L. \& CAMPBELL, J.H. 1999. Biological response to climate change on a tropical mountain. Nature 398:611-615.

SANTOS, A.J. 2003. Estimativas de riqueza em espécies. In Métodos de estudos em biologia da conservação e manejo da vida silvestre (L. Cullen Jr., R. Rudran \& C. Valladares-Pádua, orgs.). UFPR, Curitiba, p.19-41. 
SHIROSE, L.J., BISHOP, C.A., GREEN, D.M., MACDONALD, C.J., BROOKS, R.J. \& HELFERTY, N.J. 1997. Validation tests of an amphibian call count survey technique in Ontario, Canada. Herpetologica 53:312-320.

SILVANO, D.L. \& PIMENTA, B.V.S. 2003. Diversidade de anfíbios na Mata Atlântica do Sul da Bahia. In Corredor de Biodiversidade na Mata Atlântica do Sul da Bahia (P. I. Prado, E. C. Landau, R. T. Moura, L. P. S. Pinto, G. A. B. Fonseca \& K. Alger, orgs.). CD-ROM, Ilhéus, IESB/CI/ CABS/UFMG/UNICAMP.

STEBBINS, R.C. \& COHEN, N.W. 1995. Anatural History of Amphibians. Princeton University Press, New Jersey.

VASCONCELOS, T.S. \& ROSSA-FERES, D.C. 2005. Diversidade, distribuição espacial e temporal de anfíbios anuros (Amphibia, Anura) na região noroeste do Estado de São Paulo, Brasil. Biota Neotrop. 5(2): http// www.biotaneotropica.org.br/v5n $5 / \mathrm{pt} /$ abstract?article+BN01705022005 (útimo acesso em 28/ 02/2006).

YOUNG B.E., LIPS, K.R., REASER, J.K., IBÁÑEZ, R., SALAS, A.W., CEDEÑO, J.R., COLOMA, L.A., RON, S., LA MARCA, E., MEYER, J.R., MUÑOZ, A., BOLAÑOS, F., CHAVES, G. \& ROMOS, D. 2001. Population declines and priorities for amphibian conservation in Latin America. Conserv. Biol. 15:1213-1223.

Título: Diversidade e uso de habitat por anfíbios anuros em duas localidades de Mata Atlântica, no norte do estado da Bahia.

Autor: Juncá, F.A.

Biota Neotropica, Vol. 6 ( número 2 ): 2006

http://www.biotaneotropica.org.br/v6n2/pt/ abstract?inventory+bn03006022006

Recebido em 17/08/05 - Versão Reformulada recebida em 02/05/2006 - Publicado: em 20/06/2006

ISSN 1676-0603 


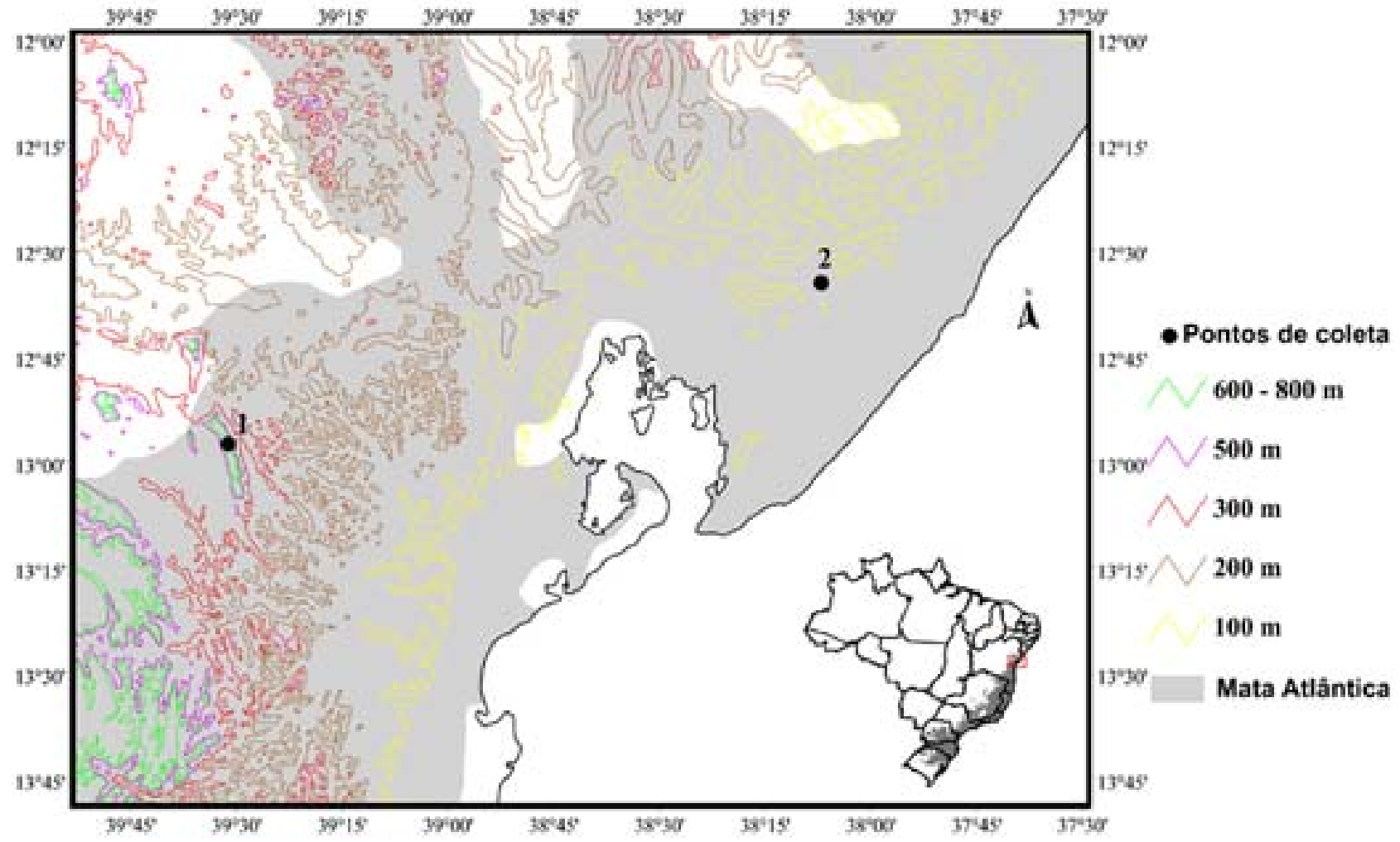

Figura 1. Localização das duas áreas amostradas no norte da Bahia, Brasil. 1 - Serra da Jibóia. 2 - Reserva Sapiranga.

Figure 1. Localization of the two sampled areas in the north of Bahia state, Brazil. 1 - Serra da Jibóia. 2 - Reserva Sapiranga. 


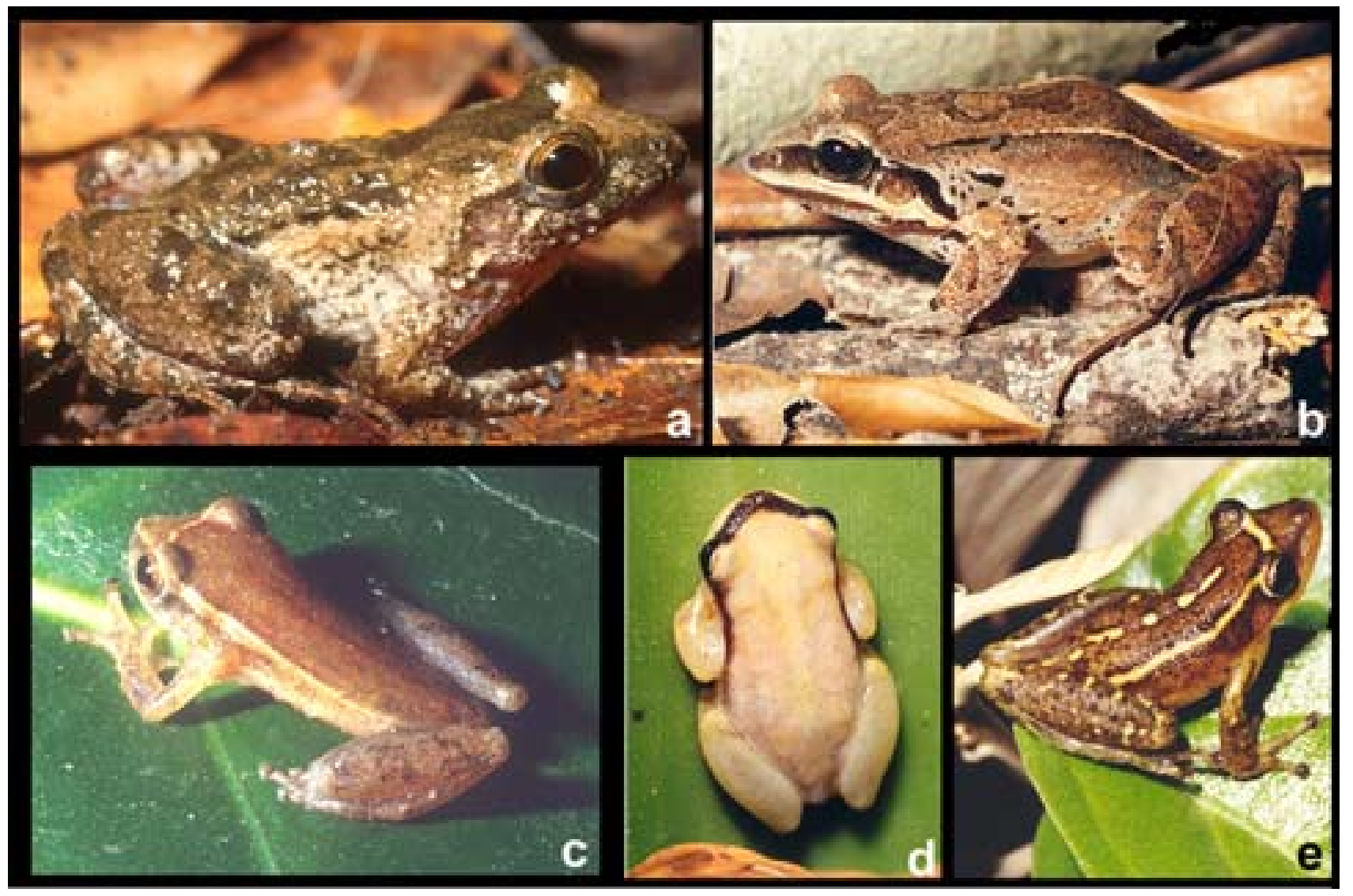

Figura 2. Espécies de anuros encontradas na Reserva Sapiranga, Bahia, Brasil. a) Leptodactylus natalensis, b) Leptodactylus mystaceus, c) Dendropsophus decipiens, $d$ ) Phyllodytes melanomystax, $e$ ) Scinax auratus.

Figure 2. Anuran species found in Reserva Sapiranga, Bahia state, Brazil. a) Leptodactylus natalensis, b) Leptodactylus mystaceus, c) Dendropsophus decipiens, $d$ ) Phyllodytes melanomystax, $e$ ) Scinax auratus. 


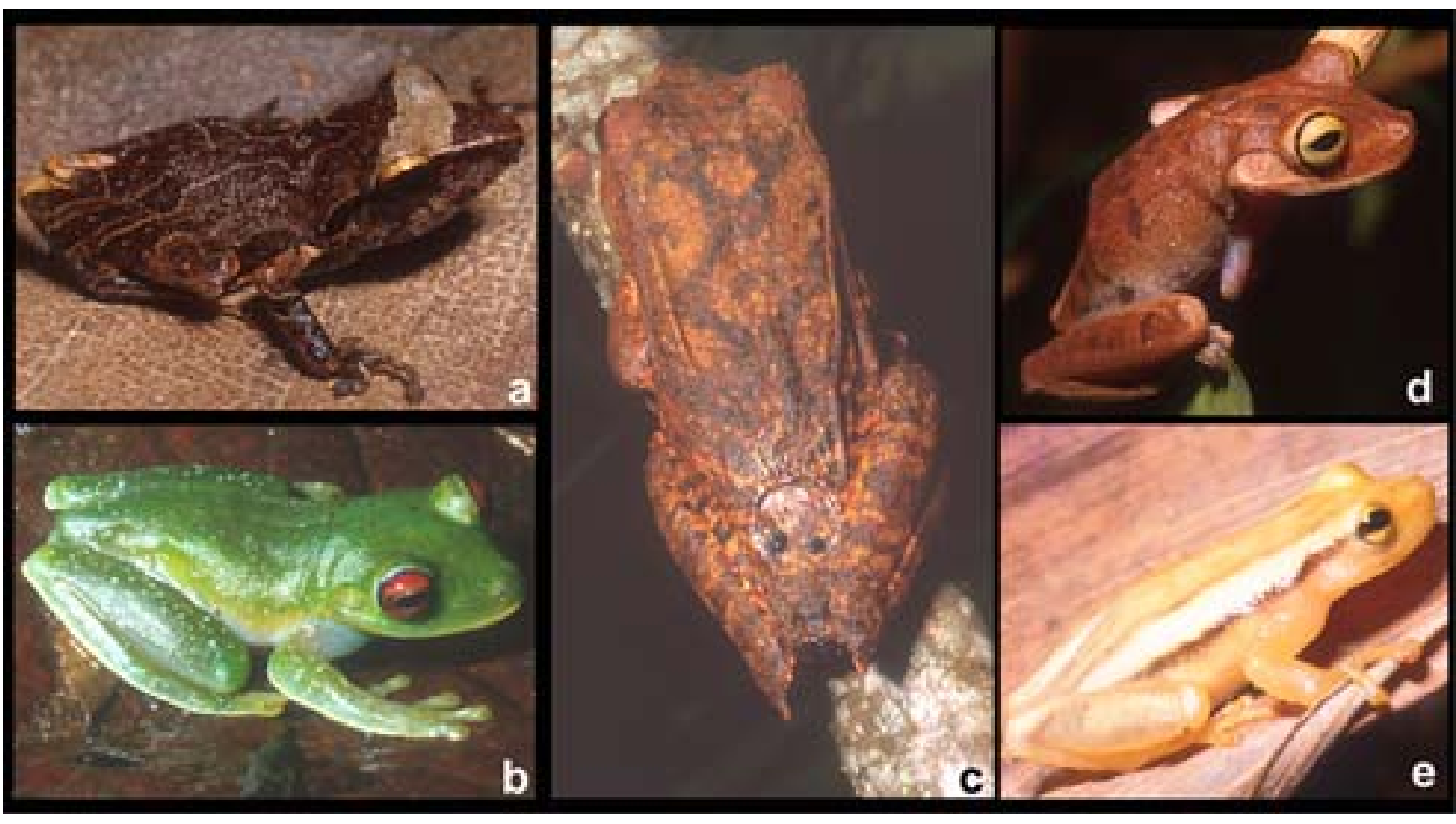

Figura 3. Espécies de anuros encontradas na Serra da Jibóia, Bahia, Brasil. a) Eleutherodactylus sp., b) Aplastodiscus sibilatus, c) Gastrotheca $s p ., d)$ Bokermannohyla hylax, $e$ ) Phyllodytes $c f$. wuchereri.

Figure 3. Anuran species found in Serra da Jibóia, Bahia state, Brazil. a) Eleutherodactylus sp., b) Aplastodiscus sibilata, c) Gastrotheca sp., d) Bokermannohyla hylax, e) Phyllodytes $c f$. wuchereri. 

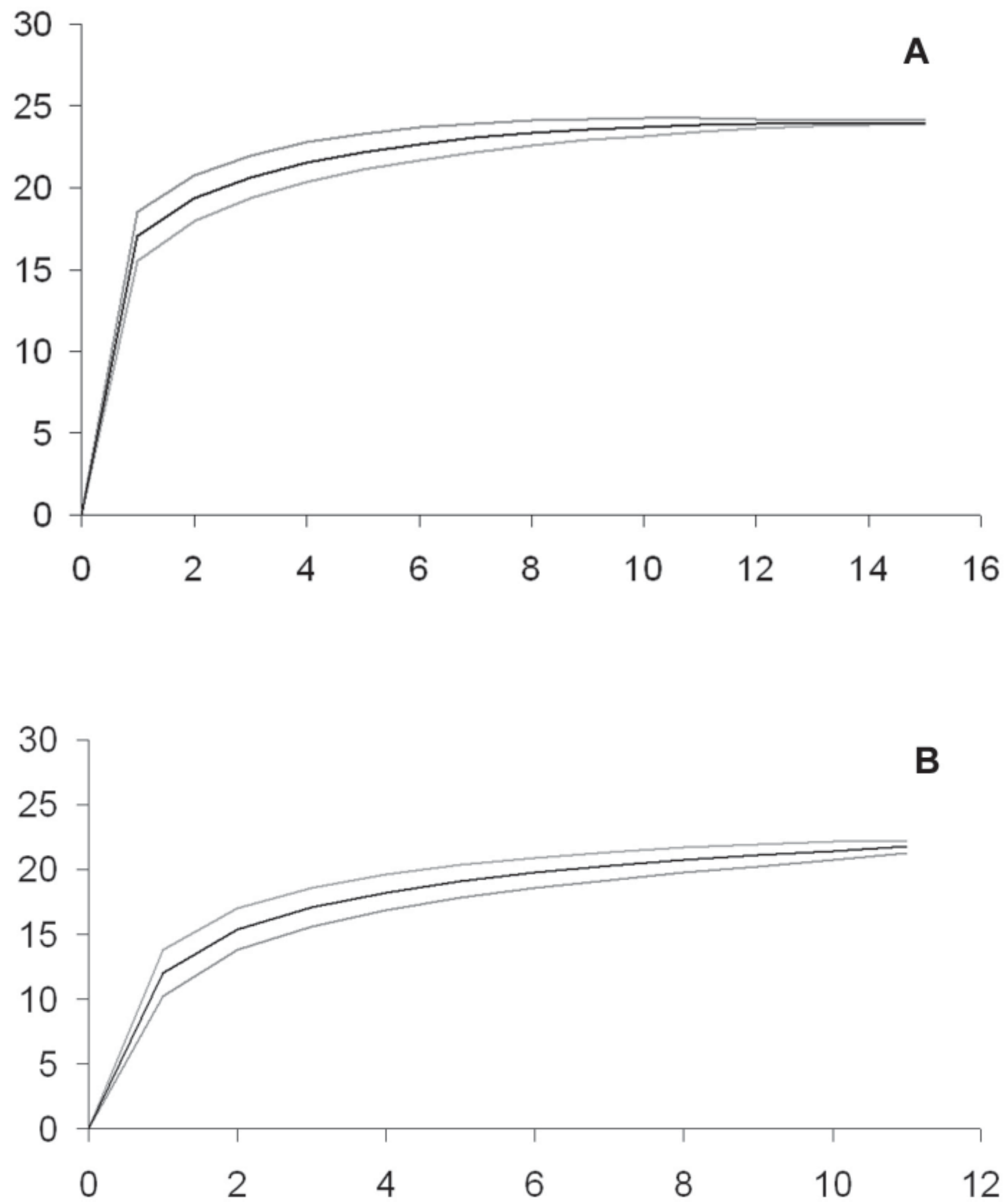

Figura 4. Curva de rarefação de espécies para duas localidades de Mata Atlântica no norte da Bahia, Brasil: A) Reserva Sapiranga (15 amostras). B) Serra da Jibóia (12 amostras). A linha preta corresponde à média obtida com 500 aleatorizações, e as linhas cinzas acima e abaixo correspondem ao desvio padrão associado.

Figure 4. Rarefaction curve of species in two localities of Mata Atlântica in the north of Bahia state, Brazil: A) Reserva Sapiranga (15 samples). B) Serra da Jibóia (12 samples). The black line is the average calculated with 500 aleatorizations and the gray lines above and below are the standard deviation.

http://www.biotaneotropica.org.br 
A Reserva Sapiranga

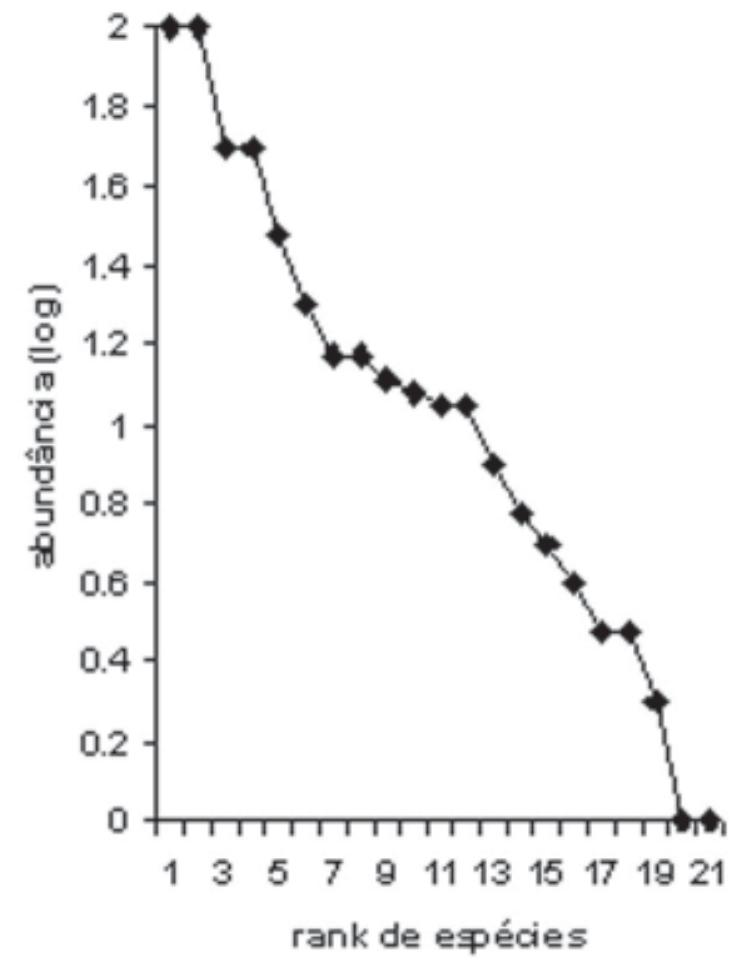

Serra da Jibóia

B

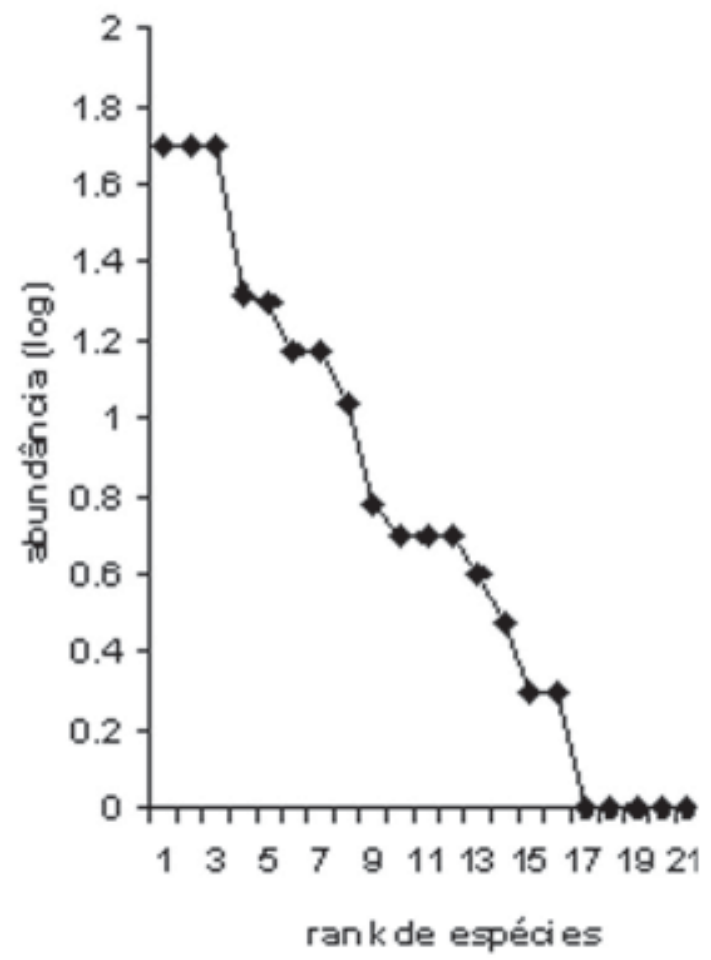

Figura 5. Curva do componente de domoninância. Reserva Sapiranga (A) e Serra da Jibóia (B).

Figure 5. Dominance-diversity curve. Reserva Sapiranga (A) e Serra da Jibóia (B). 

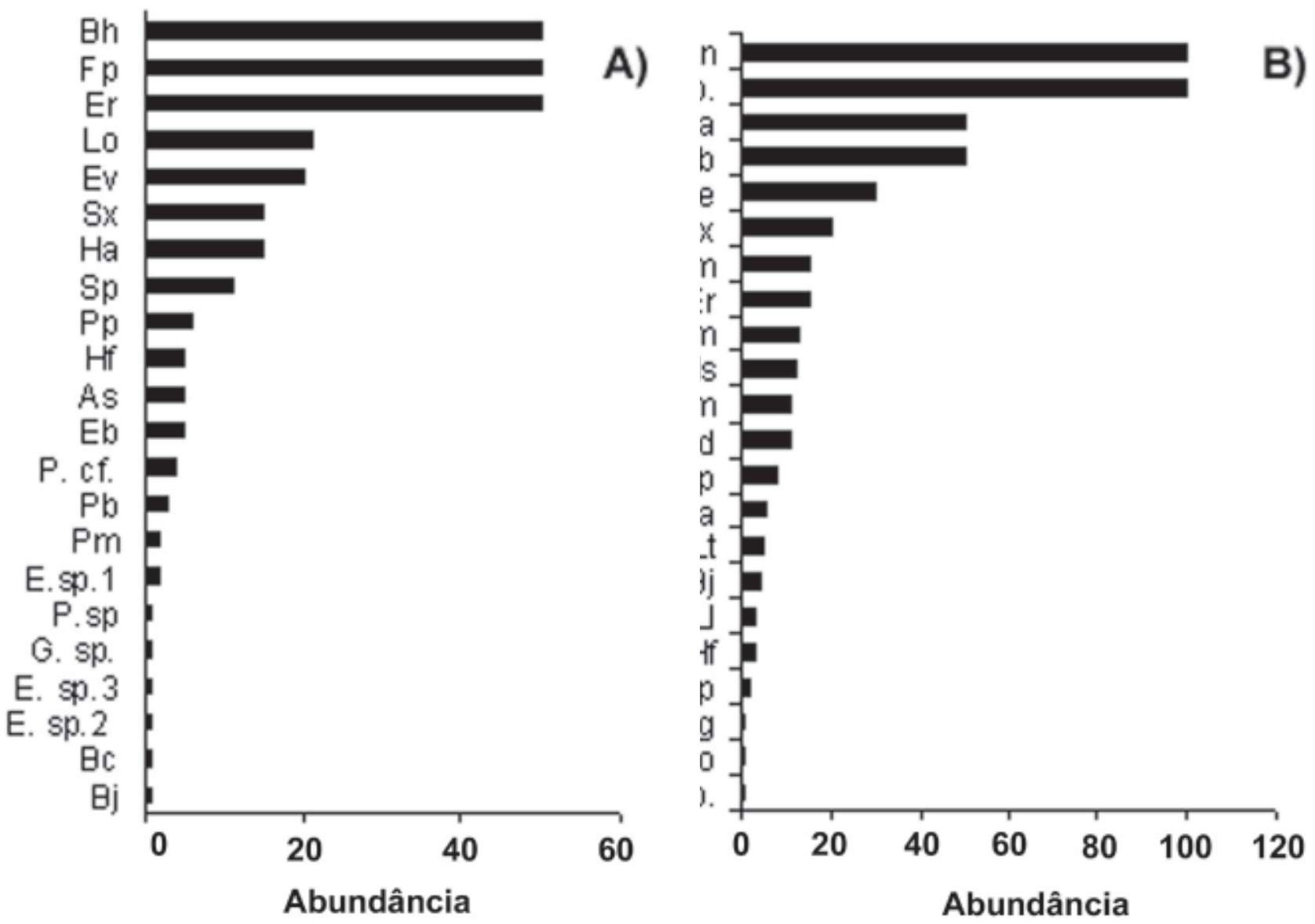

Figura 6. Abundância das espécies de anuros observadas em duas áreas de Mata Atlântica no norte da Bahia, Brasil. A) Serra da Jibóia e B) Reserva Sapiranga.

Figure 6. Abundance of anuran species observed in two Mata Atlântica areas in the north of Bahia state, Brazil. A) Serra da Jibóia e B) Reserva Sapiranga. 
Tabela 1. Esforço amostral nos diferentes hábitats.

Table 1. Sampling effort in the different habitats.

\begin{tabular}{|c|c|c|}
\hline & Serra da Jibóia & Sapiranga \\
\hline Folhedo mata & 2 transeções de $500 \mathrm{~m} \times 6 \mathrm{~m}$ & 2 transeções de $500 \mathrm{~m}$ x $6 \mathrm{~m}$ \\
\hline Folhedo campo rupestre & 2 transeções de $100 \mathrm{~m}$ x $10 \mathrm{~m}$ & Inexistente nessa área \\
\hline Bromélias de mata & Idem folhedo de mata & 1 transeção de 100 x 10 m \\
\hline Bromélias campo rupestre & 2 transeções de $100 \mathrm{~m}$ x $10 \mathrm{~m}$ & Inexistente nessa área \\
\hline Poças temporárias & $\begin{array}{l}\text { Duas poças } \\
\text { aproximadamente } 200 \mathrm{~m}^{2} \text { cada } \\
\text { uma, profundidade de até } 80 \mathrm{~cm}\end{array}$ & $\begin{array}{l}\text { Uma poça de } 300 \mathrm{~m}^{2} \text {, } \\
\text { profundidade máxima } 80 \\
\text { cm, e três poças } \\
\text { aproximadamente } 25 \mathrm{~m}^{2} \\
\text { cada uma (profundidade } 40 \\
\text { cm) }\end{array}$ \\
\hline Poças permanentes & $\begin{array}{l}\text { Duas poças } \\
\text { aproximadamente } 100 \mathrm{~m}^{2} \text { e } 30 \\
\mathrm{~m}^{2} \text { profundidade máxima } \\
\text { aproximada de } 1,5 \mathrm{~m}\end{array}$ & $\begin{array}{l}\text { Uma poça } \\
\text { aproximadamente } \\
\text { profundidade }\end{array}$ \\
\hline Riachos & $\begin{array}{l}3 \text { riachos; trecho de } 100 \\
\mathrm{~m} / \text { riacho }\end{array}$ & $\begin{array}{l}2 \text { riachos; trecho de } 80 \\
\mathrm{~m} / \text { riacho }\end{array}$ \\
\hline
\end{tabular}


Tabela 2. Ocorrência das espécies obtidas na Reserva Sapiranga e na Fazenda Camurugipe - Praia do Forte, Mata de São João, Bahia, nos cinco hábitats amostrados.

Table 2. Occurrence of species encountered in Reserva Sapiranga and Fazenda Camurugipe - Praia do Forte district, Mata de São João municipality, Bahia state, in the five sampled habitats.

\begin{tabular}{|c|c|c|c|c|c|}
\hline $\begin{array}{l}\text { Família } \\
\text { Espécie }\end{array}$ & $\begin{array}{c}\text { Folhedo e } \\
\text { sub- } \\
\text { bosque }\end{array}$ & Bromélias & $\begin{array}{c}\text { Poça } \\
\text { temporária }\end{array}$ & $\begin{array}{c}\text { Poça } \\
\text { permanente }\end{array}$ & Rio \\
\hline \multicolumn{6}{|l|}{ Leptodactylidae (9 espéc ies) } \\
\hline Eleutherodactylus ramagii & - & & & & \\
\hline Eleutherodactylus sp. (grupo binotatus) & $\bullet$ & & & & \\
\hline Leptodactylus labyrintbicus & & & • & & \\
\hline L. mystaceus & & & $\bullet$ & & \\
\hline L. natalensis & & & $\bullet$ & - & \\
\hline L. troglodytes & & & • & & \\
\hline L. ocellatus & & & $\bullet$ & & \\
\hline Physalaemus sp. & & & $\bullet$ & & \\
\hline Proceratophrys aff. boiei & & & $\bullet$ & & \\
\hline \multicolumn{6}{|l|}{ Hylidae (14 espécies) } \\
\hline Dendropsophus branneri & & & - & - & - \\
\hline D. decipiens & & & $\bullet$ & $\bullet$ & \\
\hline D. elegans & & & & $\bullet$ & \\
\hline Hypsiboas albomarginatus & & & $\bullet$ & $\bullet$ & \\
\hline H. faber & & & & $\bullet$ & \\
\hline H. pombali & & & & $\bullet$ & $\bullet$ \\
\hline H. semilineatus & & & $\bullet$ & & $\bullet$ \\
\hline Phyllodytes melanomystax & & $\bullet$ & & & \\
\hline D. minutus & & & $\bullet$ & & \\
\hline Scinax agilis & & $\bullet$ & & & \\
\hline Scinax auratus & & & $\bullet$ & $\bullet$ & \\
\hline S. $x$-signatus & & & $\bullet$ & & \\
\hline Scinax sp. (grupo ruber) & & & $\bullet$ & & \\
\hline Sphaenorbynchus prasinus & & & & • & \\
\hline \multicolumn{6}{|l|}{ Bufonidae (2 espécies) } \\
\hline Bufo jimi & & & • & $\bullet$ & \\
\hline Bufo sp. (grupo margaritifer)* & $\bullet$ & & & & \\
\hline Total & 3 & 2 & 18 & 11 & 4 \\
\hline
\end{tabular}

* Incluída na lista com base em exemplar depositado na Coleção de Animais Peçonhentos e Herpetologia do Museu de Zoologia da Universidade Estadual de Feira de Santana (MZUEFS). 
Tabela 3. Ocorrência das espécies registradas na Serra da Jibóia, Santa Terezinha e Elísio Medrado, Bahia, nos cinco tipos de ambientes amostrados nessa localidade.

Table 3. Occurrence of species encountered in Serra da Jibóia, Santa Terezinha and Elísio Medrado municipalities, Bahia state, in the five sampled habitats

\begin{tabular}{|c|c|c|c|c|c|}
\hline $\begin{array}{c}\text { Família } \\
\text { Espécie }\end{array}$ & $\begin{array}{l}\text { Folhedo e } \\
\text { sub-bosque }\end{array}$ & Bromélia & $\begin{array}{c}\text { Poças } \\
\text { temporárias }\end{array}$ & $\begin{array}{c}\text { Poças } \\
\text { permanentes }\end{array}$ & $\overline{\text { Rios }}$ \\
\hline \multicolumn{6}{|l|}{ Leptodactylidae (13 espécies) } \\
\hline Adelophryne pachydactyla & & - & & & \\
\hline Crossodactylus sp.* & & & & & - \\
\hline Eleutherodactylus bilineatus & - & & & & \\
\hline E. ramagii & $\bullet$ & & & & \\
\hline E. vinhai & $\bullet$ & & & & \\
\hline Eletutherodactvlus sp.1 (grupo & • & & & & \\
\hline \multicolumn{6}{|l|}{ binotatus) } \\
\hline Eletutherodactylus sp.2 (grupo & - & & & & \\
\hline \multicolumn{6}{|l|}{ ramagii) } \\
\hline Eleutherodactylus sp.3 & & $\bullet$ & & & \\
\hline Leptodactylus ocellatus & & & & • & \\
\hline Odontophrynus carvalboi* & • & & & & \\
\hline Physalaemus sp. (grupo cuvieri) & $\bullet$ & & & & \\
\hline Proceratophrys precrenulata & $\bullet$ & & & & - \\
\hline Thoropa miliaris* & & & & & $\bullet$ \\
\hline \multicolumn{6}{|l|}{ Hylidae (13 espécies) } \\
\hline Aplastodiscus sibilatus & & & & • & - \\
\hline Bokermannobyla bylax & & & $\bullet$ & $\bullet$ & $\bullet$ \\
\hline Gastrotheca fissipes* & & - & & & \\
\hline Gastrotheca sp.** & - & & & & \\
\hline Hypsiboas albomarginatus & & & & $\bullet$ & \\
\hline Hypsiboas crepitans* & & & & $\bullet$ & \\
\hline Hypsiboas faber & & & & $\bullet$ & - \\
\hline Phyllodytes cf. wuchereri & & - & & & \\
\hline P. melanomystax & & $\bullet$ & & & \\
\hline Phyllomedusa babiana & & & & • & \\
\hline Scinax pachycrus & & & & $\bullet$ & \\
\hline Scinax $x$-signatus & & & & • & \\
\hline \multicolumn{6}{|l|}{ Dendrobatidae (1 espécie) } \\
\hline Colostethus alagoanus* & - & & & & \\
\hline \multicolumn{6}{|l|}{ Bufonidae ( 3 espécies) } \\
\hline Bufo crucifer & - & & & & \\
\hline Bufo jimi & $\bullet$ & & & & \\
\hline Frostius pernambucensis & - & - & & & \\
\hline Total & 14 & 5 & 1 & 11 & 6 \\
\hline
\end{tabular}

* Incluída na lista com base em exemplar(s) depositado(s) na Coleção de Animais Peçonhentos e Herpetologia do Museu de Zoologia da Universidade Estadual de Feira de Santana (MZUEFS). ** Uma fêmea coletada próximo ao folhedo da mata, porém provavelmente ocorre no dossel da mata. 